\title{
Microprocessor controlled movement of liquid gastric content using sequential neural electrical stimulation
}

\author{
M P Mintchev, C P Sanmiguel, S J Otto, K L Bowes
}

\begin{abstract}
Background-Gastric electrical stimulation has been attempted for several years with little success.

Aims-To determine whether movement of liquid gastric content could be achieved using microprocessor controlled sequential electrical stimulation.

Methods-Eight anaesthetised dogs underwent laparotomy and implantation of four sets of bipolar stainless steel wire electrodes. Each set consisted of two to six electrodes $(10 \times 0.25 \mathrm{~mm}, 3 \mathrm{~cm}$ apart $)$ implanted circumferentially. The stomach was filled with water and the process of gastric emptying was monitored. Artificial contractions were produced using microprocessor controlled phase locked bipolar four second trains of $50 \mathrm{~Hz}, 14 \mathrm{~V}$ (peak to peak) rectangular voltage. In four of the dogs four force transducers were implanted close to each circumferential electrode set. In one gastroparetic patient the effect of direct electrical stimulation was determined at laparotomy.

Results-Using the above stimulating parameters circumferential gastric contractions were produced which were artificially propagated distally by phase locking the stimulating voltage. Averaged stimulated gastric emptying times were significantly shorter than spontaneus emptying times $\left(t_{1 / 2} 6.7\right.$ (3.0) versus 25.3 (12.9) minutes, $p<0.01)$. Gastric electrical stimulation of the gastroparetic patient at operation produced circumferential contractions.
\end{abstract}

Conclusions-Microprocessor controlled electrical stimulation produced artificial peristalsis and notably accelerated the movement of liquid gastric content.

(Gut 1998;43:607-611)

Keywords: gastric electrical stimulation; gastric motility

Surgery, University of

Alberta, Edmonton,

Alberta, Canada

C P Sanmiguel

S J Otto

K L Bowes

Correspondence to: Dr M P Mintchev, Department of Electrical and Computer Engineering,

University of Calgary,

Calgary, Alberta,

Canada T2N $1 \mathrm{~N} 4$

Accepted for publication 29 April 1998 voltage trains of frequency higher than $5 \mathrm{~Hz}$. This type of high frequency electrical stimulation can be called "neural electrical gastric stimulation" (NEGS). In 1963 Bilgutay et $a l^{1}$ described notable shortening of the duration of postoperative ileus in patients using NEGS with a single antral intraluminal electrode and a single cutaneous reference electrode. Subsequent well controlled studies, however, failed to confirm any significant effect of NEGS on antral contractions or postoperative ileus. ${ }^{2}{ }^{3}$

Later studies have focused on electrical control activity (ECA) entrainment, termed gastric electrical pacing (GEP) by Sama et al. ${ }^{11}$ Distal antral stimulation in dogs produced orad propagation of ECA and a delay in emptying of liquids and solids. However, proximal stimulation to entrain ECA to a higher frequency had no effect on antral emptying. These findings were confirmed by Kelly et al in $1977^{12}$ who showed retrograde propulsion of duodenal contents with distal duodenal stimulation and entrainment of the duodenal pacesetter potential. Recently, Eagon and Kelly studied carefully the effects of lower frequency $(0-20 \mathrm{~Hz})$ electrical stimulation on canine gastric electrical activity (GEA), motility and emptying, concluding that although an increment of GEA frequency was observed when stimulating at 6 and 30 cycles per minute (cpm), gastric contractions and emptying were not affected by GEP. ${ }^{6}$ However, Familoni et $a l,{ }^{8}$ in their recent investigation using a canine model of GEP, described some increased contractile activity when stimulating with frequency four to five times higher than the electrophysiological, but they did not measure gastric emptying. Another study reported diminished nausea and vomiting in patients treated with GEP, but the impact of pacing on gastric emptying remained not well clarified. ${ }^{5}$

In 1995 Chen et $a l^{13}$ described slight acceleration of gastric emptying in a pilot study of a small number of patients with gastroparesis by performing GEP at one site on the greater curvature of the stomach and entraining ECA to a frequency $10 \%$ higher than the basal. However, in 1992, Bellahsene et al had failed to show any effect from GEP in a canine model of gastroparesis. ${ }^{14}$ Surprisingly, this study showed an acceleration of gastric emptying after giving glucagon and applying GEP to five vagotomised dogs. The authors explained this finding by suggesting that GEP acts through recoordination of electromechanical events disrupted by glucagon. ${ }^{14}$ 


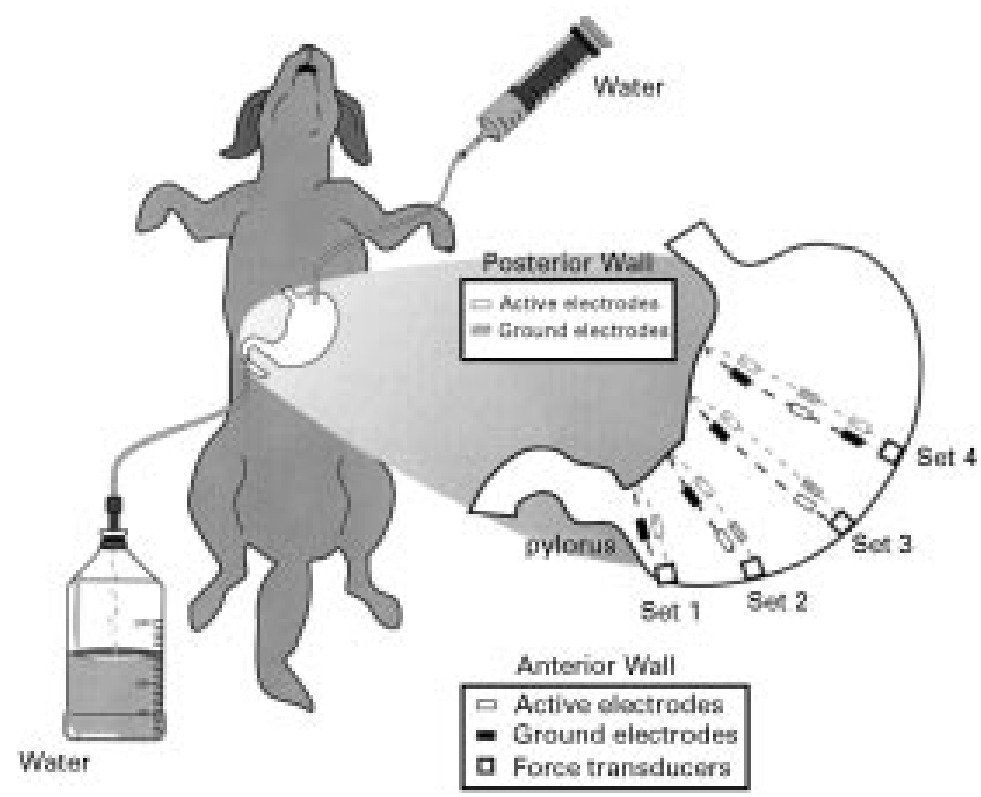

Figure 1 Schematic drawing of a canine stomach with four sets of circumferentially implanted electrodes. The active electrodes and the ground electrodes in each circumferential set were separately short circuited. The stomach was filled with 600-800 $\mathrm{ml}$ water through an intragastric tube introduced in the fundus. After initiating the sequential stimulation, movement of liquid content was monitored through an intraduodenal tube and was quantified using a scaled container.

We have previously described and tested a method for producing artificially propagated antral contractions by NEGS..$^{10}$ In the present study the effect of such stimulation on gastric emptying of liquids in an acute dog model was determined.

\section{Methods}

Eight healthy dogs with similar dimensions (five males, three females; body mass index (weight, $\mathrm{kg} /(\text { height, } \mathrm{m})^{2} 11.6(2.8) \mathrm{kg} / \mathrm{m}^{2}$; weight 18.5 (3.9) $\mathrm{kg}$ ) underwent laparotomy under Pentothal anaesthesia (Abbott, Montreal, Quebec, Canada) and implantation of locally designed bipolar stainless steel wire electrodes. The initial dosage of anaesthetic was $30 \mathrm{mg} / \mathrm{kg}$ and was supplemented with 3 $\mathrm{mg} / \mathrm{kg}$ as needed based on monitoring the restoration of the blinking effect. ${ }^{16}$ Artificial venti-

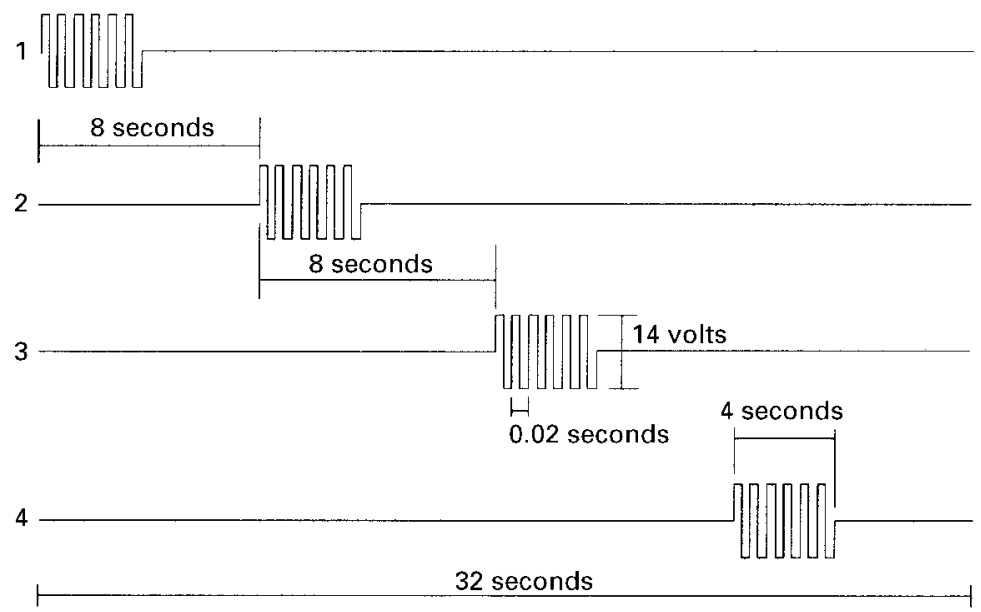

Figure 2 Time characteristics of the stimuli applied to the different circumferential electrode sets starting with the most proximal set. lation was made available during all experiments. At the end of each experiment the animal was sacrificed with an anaesthetic overdose. Each implanted electrode was a stainless steel wire $(10 \times 0.25 \mathrm{~mm})$. Four sets of electrodes were inserted at approximately 2, 6, 10, and $14 \mathrm{~cm}$ from the pylorus. Each set consisted of two (the most distal set) to six isolated electrode wires (the most proximal set) with $1 \mathrm{~cm}$ metal tips. The tips were inserted and sutured subserosally in a circumferential fashion (fig 1). The interelectrode distance in each set was between 2.5 and $3.5 \mathrm{~cm}$ depending on the gastric circumference in the given location. Every alternative electrode from a given electrode ring was connected to a common ground. All four sets of wires were connected to a four channel microprocessor controlled digital stimulator (four channel, 12 bit digital to analogue converter with up to $6.5 \mathrm{~mA}$ current output per channel, controlled by specially designed software on an IBM 486-33 personal computer).

In four of the dogs four force transducers (RB Products, Madison, Wisconsin, USA) were implanted close to each circumferential electrode set by suturing each end of the sealed unit to the gastric serosa. Recordings from the force transducers were performed using a Beckman 611 multichannel recorder (Allendale, New Jersey, USA). Considering the potential impact of external factors related to the transducer attachment to the serosa, only relative measurements of the spontaneous and the artificially invoked force of contractions were performed. ${ }^{11}$ The amplitudes of the force transducer recordings before and during stimulation were examined and the differences in their ranges were compared.

Computer modelling based on the previously described conoidal dipole model of gastric electrical activity indicated that propagated contractions could be produced circumferentially using four rings of stimulating electrodes implanted along the gastric circumference and supplied simultaneously with phase locked bipolar trains of $50 \mathrm{~Hz}, 15 \mathrm{~V}$ (peak to peak) rectangular voltage ${ }^{15}$ and interval between one stimulus and the next of 16 seconds. In the present study we increased the interval between the stimuli to 32 seconds and reduced peak to peak amplitude of the stimulating trains to $14 \mathrm{~V}$ (fig 2) in order to achieve lower energy consumption and lessen the requirements for the future implantable electronic pacemaker. A software package was developed for real time control of the multichannel digital to analogue converter using TurboC++ v.3.0 programming language (Borland Inc., Scotts Valey, California, USA). The software performed the following functions in real time: (1) displayed a conoidal computer model of the stomach with the approximate positions of the stimulating circumferential electrodes; (2) digitally synthesised and displayed the applied stimuli; (3) controlled the stimulation delivering outputs of the digital to analogue converter; and (4) showed local deformations on the conoidal wall of the modelled stomach whenever the synthesised stimuli 


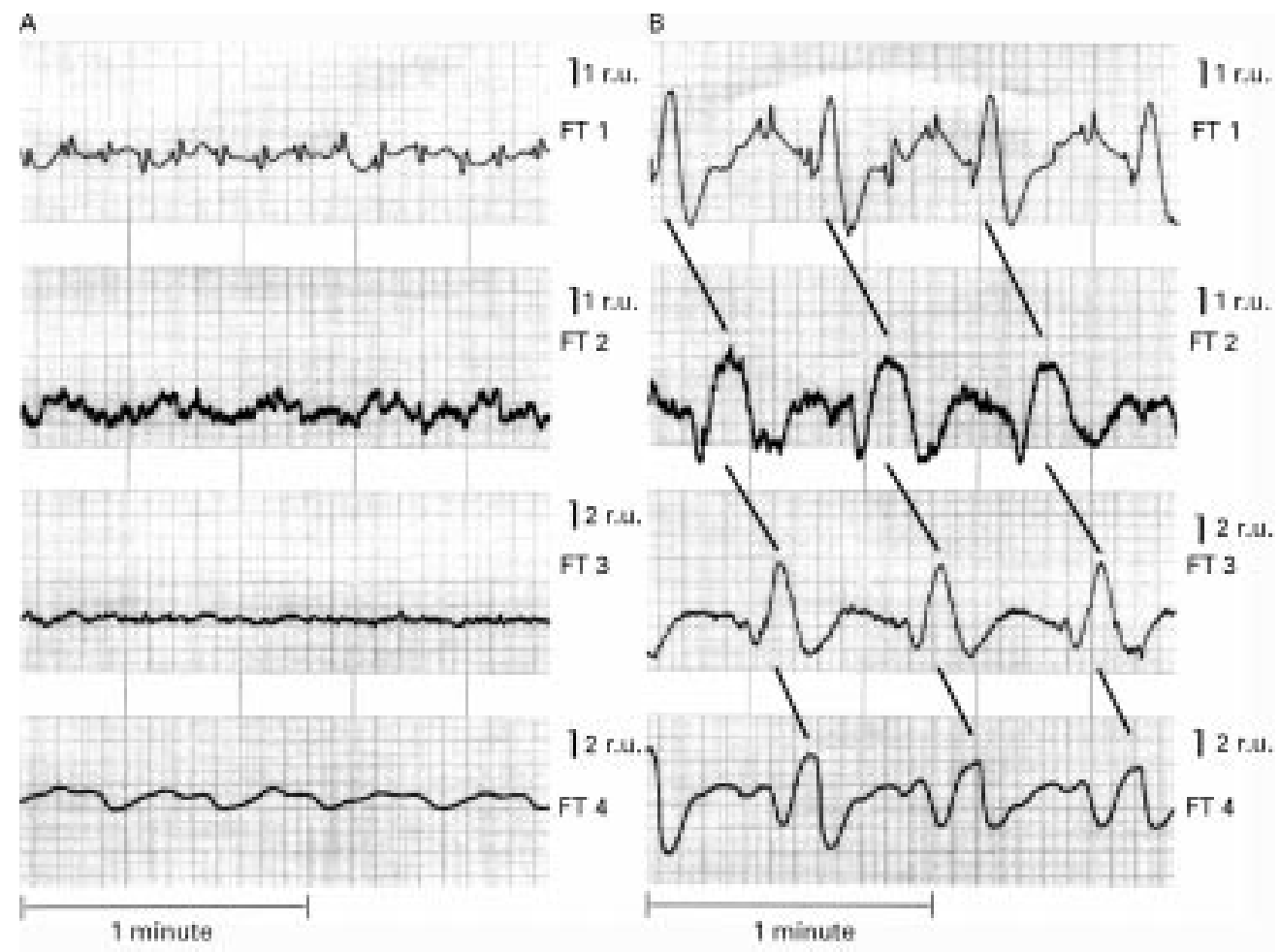

Figure 3 Gastric contractions without stimulation $(A)$ and with stimulation $(B)$ registered with force transducers (FT1-FT4). The amplitudes of the recordings were measured in relative units (r.u.).

were applied to the respective circumferential electrode set.

Stimulated and spontaneous gastric emptying of liquid content were compared. A large bore plastic tube (diameter $0.5 \mathrm{~cm}$ ) was introduced into the apex of the gastric fundus in order to fill the stomach with water. Another tube (diameter $1.5 \mathrm{~cm}$ ) was positioned in the descending duodenum and the duodenum occluded distal to it. The stomach was filled with 600 or $800 \mathrm{ml}$ water (the smaller volume was used for dogs with smaller dimensions). We compared the times to empty $200 \mathrm{ml}$ water (the estimated amount in the antrum) with and without stimulation. After each emptying session $200 \mathrm{ml}$ water was added to the stomach so that the volume of water in the stomach remained the same before each measurement. The tests were repeated at random three times per dog. The results for the emptying times were averaged and a single mean value and its standard deviation was obtained for each dog. The two sets of half emptying times $\left(t_{1 / 2}\right.$ obtained using stimulation, and through spontaneous emptying) were statistically examined using a standard $\chi^{2}$ test for significance ${ }^{16}$ with the spontaneous emptying mean half times being the expected values.

The canine experiments were approved by the Animal Welfare Committee at the Faculty of Medicine, University of Alberta.

In addition, a written consent to test the ability to produce circumferential contractions artificially on gastroparetic stomach was obtained from a 32 year old female patient diagnosed with severe gastroparesis who was undergoing laparotomy and gastrectomy. A set of four circumferential electrodes (two active and two ground) similar to set no 3 from fig 1 used in the canine experiments was implanted about $8-10 \mathrm{~cm}$ proximal to the pylorus and a stimulating voltage with the characteristics shown in channel 3 from fig 2 was applied. As the patient was about to undergo total gastrectomy and this new method of pacing is still in its infancy, we aimed at testing our ability to produce strong sequential contractions in a case of severe gastroparesis. The circumferential electrodes were not implanted permanently and gastric emptying tests were not performed on this patient. The artificially produced contractions were evaluated only visually. The experiment was approved by the Ethics Committee at the Faculty of Medicine, University of Alberta.

\section{Results}

GASTRIC MOTILITY MEASUREMENTS

Using $14 \mathrm{~V} / 50 \mathrm{~Hz}$ rectangular trains, each of duration of four seconds followed by a four second pause (fig 2), we were able to produce clearly seen gastric contractions which were artificially propagated distally by phase locking the stimulating voltage. The duration of these artificial contractions was about eight seconds after the high frequency pulses were applied, with a relaxation time of approximately four seconds and a time shift between the contractions in successive channels completely synchronous with the start of the artificial stimulation in the given electrode set (also about eight seconds). The strength of these invoked contractions was three to five times higher than the strength of the spontaneous 
Table 1 Effect of electrical stimulation on gastric emptying of $200 \mathrm{ml}$ water

\begin{tabular}{lll}
\hline & $\begin{array}{l}\text { Spontaneous emptying } t_{1 / 2} \\
(\text { min) mean }(S D)\end{array}$ & $\begin{array}{l}\text { Stimulated emptying } t_{1 / 2} \\
(\text { min) mean }(S D)\end{array}$ \\
\hline Dog 1 & $38.6(4.2)$ & $2.7(0.4)$ \\
Dog 2 & $22.6(3.1)$ & $4.0(1.0)$ \\
Dog 3 & $48.4(6.8)$ & $9.4(1.9)$ \\
Dog 4 & $31.3(3.2)$ & $4.7(0.3)$ \\
Dog 5 & $16.5(18.4)$ & $9.3(4.0)$ \\
Dog 6 & $13.3(1.2)$ & $11.3(7.2)$ \\
Dog 7 & $19.3(6.8)$ & $6.8(2.3)$ \\
Dog 8 & $12.3(5.13)$ & $5.6(1.15)$ \\
\hline
\end{tabular}

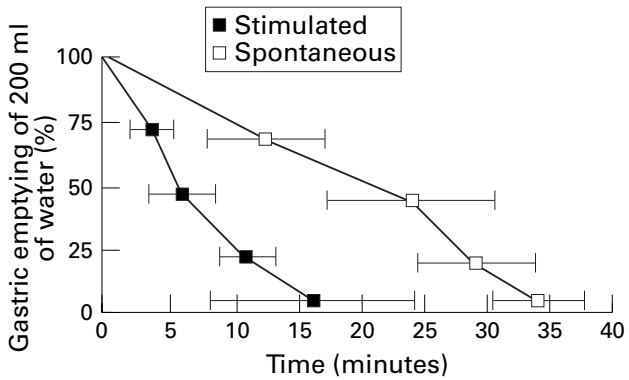

Figure 4 Spontaneous and stimulated gastric emptying of $200 \mathrm{ml}$ water in the canine model. A substantial increment in gastric emptying during stimulation was noted.

contractions (fig 3). The total stimulating current drawn from each electrode set increased gradually in the proximal direction from approximately $1-1.5 \mathrm{~mA}$ (for the most distal set) to $6-6.5 \mathrm{~mA}$ (for the most proximal set).

Using stimulating parameters similar to the ones from fig 2 (channel 3), we were able to produce visibly strong circumferential contractions in the stomach of the gastroparetic patient.

GASTRIC EMPTYING MEASUREMENTS

The invoked artificially propagated circumferential contractions moved liquid content into the duodenum synchronously with the period of repetition of the stimulating trains (32 seconds, see fig 2). Stimulated mean half emptying times for each dog were significantly lower than spontaneous mean half emptying times $(p<0.01$; table 1$)$. The averages of overall mean half times for gastric emptying of water were 25.28 (12.9) minutes without stimulation and 6.72 (3.0) minutes with stimulation. Figure 4 shows a typical example of gastric emptying rate with and without electrical stimulation.

A typical contractile pattern during the periods of spontaneous emptying (without any stimulation) is shown in fig $3 \mathrm{~A}$. Artificially invoked and synchronised contractions which produced the invoked emptying are shown in fig 3B.

\section{Discussion}

The report of Bilgutay et al in $1963^{1}$ aroused considerable interest in possible applications of electrical stimulation for the treatment of gastroparesis and other related motility disorders. The two possible mechanisms by which electrical stimulation might affect gastric emptying are: NEGS, produced when stimulating with relatively high frequencies; and GEP, which attempts to improve the entraining and synchronisation of ECA using frequencies close to or slightly higher than the electrophysiological frequency of the stomach. GEP has unfortunately has not proved to be as an effective method for the acceleration of gastric emptying. ${ }^{5}{ }^{13}$

In this study we applied microprocessor controlled sequential NEGS with four second $50 \mathrm{~Hz} / 14 \mathrm{~V}$ bipolar rectangular voltage trains and the canine smooth muscle responded with strong contractions without visible damage of the tissue surrounding the implanted electrodes. This approach could be regarded as a rough recreation of the natural gastric "microprocessor". Our previous study indicated that this type of stimulation is largely mediated through cholinergic neural pathways. ${ }^{10} 15$

One of the major problems in the use of NEGS is the production of propagated contractions. ${ }^{6}{ }^{15}$ We addressed this issue by using four circumferential electrode sets positioned 3-5 $\mathrm{cm}$ apart in the distal half of the stomach. By phase locking the applied stimuli between the successive electrode sets (starting from the most proximal set) we were able to propagate artificially the contraction distally. This propagation was controlled by a microprocessor and monitored with force transducers. Movement of liquid gastric content into the duodenum was synchronous with the period of the stimulating trains. Movement of liquid gastric content was dramatically accelerated by this artificial method of pacing $(\mathrm{p}<0.01)$. The flexibility of the stimulation and synchronisation patterns, and of the durations of stimulation and rest achieved by the microprocessor based control of the stimuli delivering digital to analogue converter makes this stimulation method easily adjustable to different patterns of stimulation and rates of gastric emptying that might be needed in patients.

A separate study of one patient indicated that at least in some patients with severe gastroparesis, high frequency NEGS can produce circumferential contractions, and gastric electrical stimulation using this method may be applicable clinically. However, careful morphological studies are needed to investigate the effect of this pacing method on the smooth muscle cells surrounding the implanted electrodes, and more studies are required to investigate gastric emptying of liquids and solids in acute and chronic animals, and the long term effects of sequential NEGS before applying this methodology to humans.

The approach suggested in this paper is still in its infancy, and was tested on anaesthetised animals only. Whether this artificial microprocessor controlled "massaging" of the stomach is not in fact quite painful is still to be determined in chronic experiments. Much attention should be paid to the possible tissue damage after prolonged stimulation. In addition, we used a duodenal tube to show the propagation of liquid to the duodenum, while in chronic experiments the distal duodenal resistance to flow might be an important additional factor that would disturb the process of artificial emptying. However, we believe that the present work shows the visibility of the artificial microproc- 
essor based recreation of gastrointestinal motor function and envisage a future in which the design of implantable microprocessor based gastrointestinal stimulators would be possible.

The technology described in this paper is protected by a pending patent. The work was supported in part by grants from Whitaker Foundation and Natural Sciences and Engineering Research Council of Canada.

1 Bilgutay AM, Wingrove R, Griffin WO, et al. Gastrointestinal pacing. A new concept in the treatment of ileus. Ann Surg 1963;158:338-43.

2 Quast DC, Beall AC, DeBakey ME. Clinical evaluation of the gastrointestinal pacer. Surg Gynec Obstet 1965;120:35-40.

3 Berger T, Kewenter J, Kock NG. Response to gastrointestinal pacing: antral, duodenal and jejunal motility in control and postoperative patients. Ann Surg 1966;161: 139-44.

4 Miedema BW, Sarr MG, Kelly KA. Pacing the human stomach. Surgery 1992;111:143-50.

5 The GEMS Group. Electrical stimulation for the treatment of gastroparesis - preliminary report of a multicenter interor gastroparesis - prelininary report of a multicenter inter-

6 Eagon JC, Kelly KA. Effect of electrical stimulation on gastric electrical activity, motility and emptying. Neurogastroentric electrical activity, mo
terol Motil 1995;7:39-45.

7 Hocking MP, Vogel SB, Sininsky CA. Human gastric myoelectric activity and gastric emptying following gastric sur-
8 Familoni BO, Abell TL, Nemoto D, et al. Efficacy of electrical stimulation at frequencies higher than basal rate in canine stomach. Dig Dis Sci 1997;42:892-7.

9 Christensen J. Responses of the smooth muscle segment of the opossum esophagus to distention and electrical stimulation, and their modification by antagonist. In: Gastrointestinal Motility. International Symposium on Motility of the Gastrointestinal Tract. 1969 July 15-16. Erlangen, 1969: 167-74.

10 Mintchev MP, Bowes KL. Production of propagated antral contractions by electrical stimulation. Dig Dis Sci 1996;41: 1890.

11 Sama SK, Bowes KL, Daniel EE. Gastric pacemakers. Gastroenterology 1976;70:226-31.

12 Kelly KA, Code CF. Duodenal-gastric reflux and slowed gastric emptying by electrical pacing of the canine duodenal pacesetter potential. Gastroenterology 1977;72: 429.

13 Chen JDZ, Lin ZY, Schirmer BD, et al. Effect of gastric pacing with optimal parameters on gastric emptying in patients with gastroparesis. In: Proceedings of XV International Symposium on Gastrointestinal Motility. 1995 Oct 21-28; Rome, Italy. 1995:42.

14 Bellahsene BE, Lind CD, Schirmer BD, et al. Acceleration of gastric emptying with electrical stimulation in canine model of gastroparesis. Am f Physiol 1992;262:G826-34.

15 Mintchev MP, Bowes K-L. Computer model of gastric electrical stimulation. Ann Biomed Eng 1997;25:726-30.

16 Mintchev MP, Otto SJ, Bowes KL. Electrogastrography can recognize gastric electrical uncoupling in dogs. Gastroenterology 1997;112:2006-11. 